\title{
Frontal Assessment Battery to Evaluate Frontal Lobe Dysfunction in ALS Patients
}

\author{
Suk-Won Ahn, Su-Hyun Kim, Jee-Eun Kim, Sung-Min Kim, Seung Hyun Kim, \\ Jung-Joon Sung, Kwang-Woo Lee, Yoon-Ho Hong
}

\begin{abstract}
Background: Assessment of frontal lobe impairment in amyotrophic lateral sclerosis (ALS) is a matter of great importance, since it often causes ALS patients to decrease medication and nursing compliance, thus shortening their survival time. Methods: The frontal assessment battery (FAB) is a short and rapid method for assessing frontal executive functions. We investigated the applicability of the FAB as a screening method for assessing cognitive impairments in 61 ALS patients. Depending on the results of the FAB, we classified patients into two subgroups: FAB-normal and FAB-abnormal. We then performed additional evaluations of cognitive function using the Korean version of the mini-mental state examination (K-MMSE), a verbal fluency test (COWAT), and a neuropsychiatric inventory (NPI). Results of these tests were compared between the two groups using Mann-Whitney U-tests, and Spearman correlation analyses were used to investigate the relationships between FAB score and disease duration and severity. Results: Of the 61 sporadic ALS patients included in this study, 14 were classified as FAB-abnormal and 47 were classified as FAB-normal. The FAB-normal and FAB-abnormal patients performed significantly differently in all domains of the COWAT. There was no difference in behavioral disturbance, as assessed by the NPI, between the two groups. The FAB scores were found to significantly correlate with both disease duration and severity. Conclusions: The FAB shows promise as a method of screening for frontal lobe dysfunction in ALS, as it is not only quick and easy, but also reliable. Additional studies should examine how FAB performance changes as ALS progresses.
\end{abstract}

RÉSUMÉ: Batterie de tests d'évaluation frontale pour la dysfonction du lobe frontal chez les patients atteints de SLA. Contexte : L'évaluation de l'atteinte du lobe frontal dans la sclérose latérale amyotrophique est très importante parce qu'elle est souvent en cause chez les patients atteints de SLA qui réduisent leur prise de médicaments et leur fidélité aux soins infirmiers, écourtant ainsi leur survie. Méthode : La batterie d'évaluation frontale (BEF) est une méthode courte et rapide d'évaluation des fonctions exécutives frontales. Nous avons étudié l'applicabilité de la BEF comme méthode de dépistage de l'atteinte cognitive chez 61 patients atteints de SLA. Selon les résultats de la BEF, nous avons divisé les patients en 2 sous-groupes, selon que la BEF était normale ou anormale. Nous avons ensuite procédé à des évaluations plus poussées de la fonction cognitive au moyen de la version coréenne de l'examen MMSE (K-MMSE), un test de fluidité verbale (COWAT) et un inventaire neuropsychiatrique (NPI). Les résultats de ces tests ont été comparés entre les deux groupes au moyen du test-U de Mann-Whitney et des analyses de corrélation de Spearman pour étudier les relations entre les scores de la BEF et la durée de la maladie et sa sévérité. Résultats : Parmi les 61 cas sporadiques de SLA inclus dans notre étude, 14 ont été classifiés comme ayant une BEF anormale et 47 une BEF normale. Les résultats dans tous les domaines du COWAT étaient significativement différents entre les patients des deux groupes. Il n'y avait pas de différence dans la perturbation du comportement entre les deux groupes, telle qu'évaluée par le NPI. Les scores de la BEF étaient corrélés de façon significative à la durée et à la sévérité de la maladie. Conclusions : La BEF est une méthode de dépistage prometteuse de la dysfonction du lobe frontal dans la SLA, non seulement parce qu'elle est rapide et facile, mais aussi parce qu'elle est fiable. Il faudra étudier comment la performance aux tests de la BEF change à mesure que la SLA progresse.

Can J Neurol Sci. 2011; 38: 242-246

Amyotrophic lateral sclerosis (ALS), the most common adult-onset motor neuron disease, is pathologically characterized by the progressive loss of upper and lower motor neurons from the brainstem motor nuclei, anterior horn of the spinal cord, and cerebral cortex. This pattern of neurodegeneration produces progressive weakness, muscular wasting, and spasticity. These begin segmentally before becoming widespread and resulting in death from respiratory failure or infection within several years of disease onset. Recent studies on ALS suggest that there are structural and pathologic changes that extend beyond the motor neurons and that some of these changes are correlated with cognitive dysfunction. For instance, some ALS patients with cognitive impairments have exhibited signs of frontal lobe deterioration..$^{1-3}$ Additionally, a number of studies have suggested an overlap between ALS and frontotemporal dementia
(FTD), and have shown that the presence of FTD in ALS patients is predictive of shorter survival time. ${ }^{4}$ Thus, early identification of frontal lobe dysfunction or FTD is essential for

From the Department of Neurology (SWA), Chung-Ang University Hospital, ChungAng University College of Medicine; Department of Neurology (SuHK, JEK, SMK, JJS, KWL), Seoul National University Hospital; Department of Neurology (YHH), Boramae Medical Center, Seoul National University College of Medicine; Department of Neurology (SeHK), Hanyang University College of Medicine, Seoul, Republic of Korea.

Received May 20, 2010. Final Revisions Submitted September 24, 2010. Correspondence to: Yoon-Ho Hong, Department of Neurology, Boramae Medical Center, Seoul National University College of Medicine, 425 Sindaebang-dong, Dongjak-gu, Seoul, 156-707, Republic of Korea. 
improving clinical care of ALS patients, as the presence of these conditions will affect decisions regarding the management of major problems and the design of medical treatment.

Identification of FTD could be facilitated by a time-efficient and diagnostically reliable measurement tool for assessing cognitive impairments. ${ }^{3}$ The frontal assessment battery (FAB) is a simple ten minute test that was designed to evaluate executive functions that are thought to be organized within the frontal lobe; this test can easily be administered at a patient's bedside or in polyclinics. ${ }^{5,6}$ The FAB was originally proposed as a valid and reliable tool for detecting executive impairment associated with neurodegenerative diseases such as Parkinson's disease, frontotemporal dementia, and Alzheimer's disease. ${ }^{7,8}$ Given the similarity between these conditions and ALS, it seems likely that FAB could be a sensitive screening tool for assessing cognitive impairment in ALS patients.

Here, we performed a variety of cognitive evaluations in 61 ALS patients in order to see whether the FAB can be used to assess frontal lobe impairments in ALS patients. Depending on their performance on the FAB, patients were divided into two groups (abnormal versus normal) and then further tested with the controlled oral word association test (COWAT), the Korean version of the mini-mental status examination (K-MMSE), and the neuropsychiatric inventory (NPI). We then evaluated whether the two groups performed statistically differently on the additional tests (in order to assess the ability of the FAB to determine whether patients had cognitive impairment), and tested for correlations between FAB and disease duration and severity (in order to evaluate whether cognitive function deteriorates with disease progression).

\section{MeTHODS}

\section{Patients}

A total of 61 participants were recruited from the ALS Clinic of the Seoul National University Hospital (a tertiary referral center) after being diagnosed with definite or laboratory- supported probable ALS, as per the revised El Escorial criteria. ${ }^{9}$ All patients provided informed consent prior to participating in the study. Patients were excluded from the study if they had a history of brain injury, stroke, alcohol or drug abuse, or recent (within one month) medication with a psychiatric drug, or if they were currently experiencing depression or major psychiatric disease. Patients were also excluded if either dysarthria (inability to repeat spoken and written words) or arm weakness (muscle strength $\leq$ Medical Research Council Grade III) were severe enough to impair performance on neuropsychological tests.

The mean disease duration, which is generally the patient's estimate of the length of time that has elapsed since onset of the first symptoms, was 16.8 months. Mean patient age was 54.5 (range: 20-79 years-old), and 42 of 61 (69\%) study participants were male. On average, patients had received 10.8 years of schooling (range: 0-18 years). Functional impairment due to ALS was evaluated using the revised amyotrophic lateral sclerosis rating scale (ALSFRS-R), which consists of 12 items measuring bulbar, upper extremity, lower extremity, and respiratory functions; scores range from 0 (severe impairment) to 48 (normal functioning). ${ }^{10}$

\section{Neuropsychological tests}

Executive functions were evaluated using the FAB, which consists of six subtests, each of which examines one of the processes controlled by the frontal lobes, including conceptualization, mental flexibility, motor programming and executive control of action, resistance to interference, selfregulation, inhibitory control, and environmental autonomy. ${ }^{6}$ The content of the subtests includes abstract reasoning on similarities, lexical fluency, Luria's "fist-edge-palm" motor series, conflicting instruction, go-no-go tasks, and prehension behavior. ${ }^{6,7}$ Each subtest is scored on a scale of $0-3$, where a score of 0 is given when the subject fails to provide an answer or responds inappropriately. These scores are added to calculate the total FAB score, which ranges from 0 to 18 . Administration of
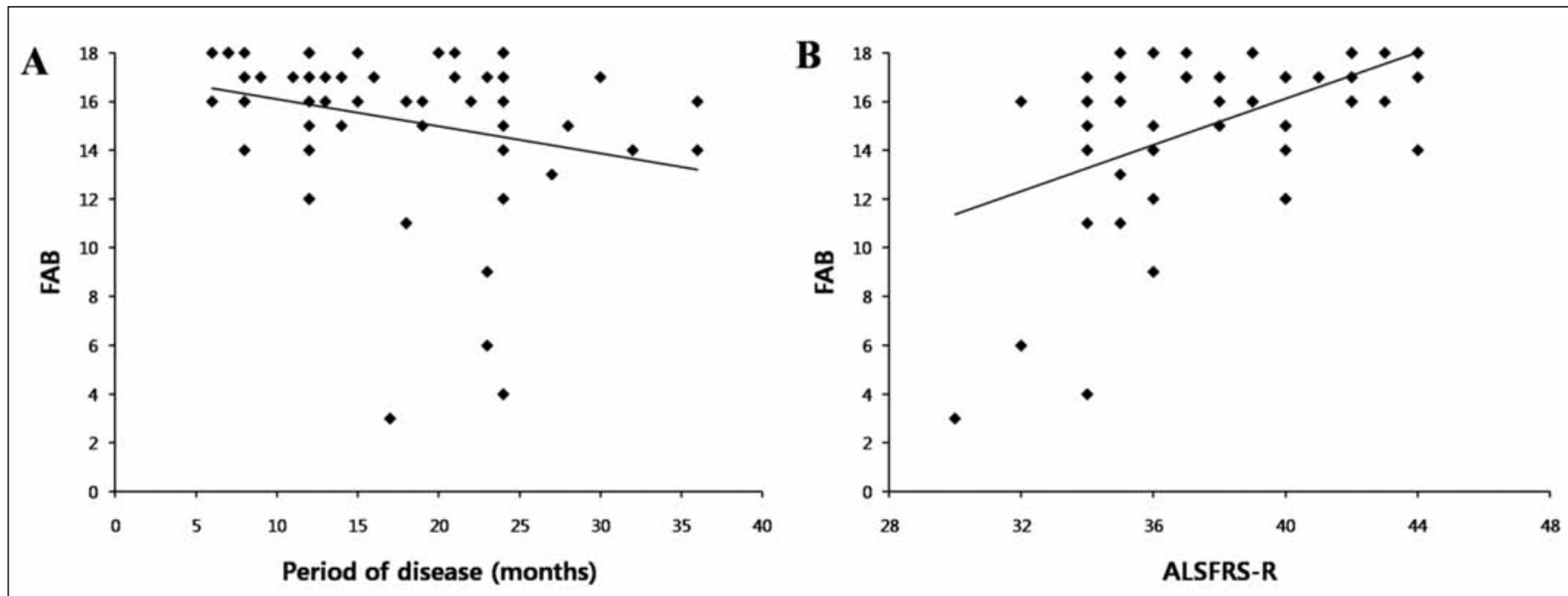

Figure: Scatter plots illustrating the correlation between the FAB scores and both disease duration (A) and ALSFRS-R (B). 
the entire battery requires approximately ten minutes. ${ }^{6,7}$ Because age and education level (years of schooling) have significant effects on performance, original scores were adjusted using the following, previously-reported formula: Adjusted FAB score = raw FAB score $-1.43 \times[\log (100-$ age $)-3.65]-0.98 \times$ [square root (years of education) -3.15$]$. $^{7}$

Verbal fluency was also assessed using the COWAT, in which patients were required to name or write as many Korean words as possible that begin with the "s" and " $k$ " sounds (spoken phonological fluency) and with the "b" and " $z$ " sounds (written phonological fluency). Patients were also requested to name as many animal and food names (spoken semantic fluency), and flower and grocery items (written semantic fluency) as possible. ${ }^{11}$

Patients' general cognitive functions were assessed using the K-MMSE. ${ }^{12,13}$ Behavioral disturbance was evaluated using the NPI, a caregiver questionnaire designed to document the presence and extent of 12 neuropsychiatric domains commonly found in dementia, including delusions, hallucinations, agitation/aggression, depression, anxiety, apathy, irritability, euphoria, disinhibition, aberrant motor behavior, night time behavior disturbances, and appetite and eating abnormalities. Caregivers who live with patient full-time (e.g., spouses, children, parents, etc.) were requested to rate the severity of each domain (maximum score $=3$ ). ${ }^{14}$
With the exception of the NPI, all tests were administered by a psychologist during the study period.

\section{Statistical analysis}

Patients were classified into two groups according to their performance on the FAB test. An adjusted FAB score of 14.11 was used as a cutoff point to classify patients as "FAB-normal" (>14.11) and "FAB-abnormal" $(<14.11)^{7}$ Mann-Whitney $U$ tests were used to compare the results of the K-MMSE, COWAT, and NPI between these two groups. Spearman correlation analyses were used to investigate the relationships between adjusted FAB scores and the duration and severity of disease. All tests were conducted in SPSS v. 17.0. Significance was defined as $P<0.05$.

\section{Results}

Of the 61 sporadic ALS patients included in this study, 14 were classified as FAB-abnormal and 47 were classified as FABnormal. ${ }^{7}$ Demographic and clinical data, including age, sex, education level, disease duration and severity, and FAB total and sub-scores are summarized in the Table.

Among the FAB subtests, the most difficult appeared to be the lexical fluency (39 patients), go-no-go (25 patients), and conflicting instruction (17 patients) tasks. By contrast, many patients performed well on the subtests examining prehension

Table: Clinical characteristics of ALS patients who were categorized according to FAB results, and comparisons of FAB, K-MMSE, COWAT, and NPI between FAB-normal and FAB-abnormal groups

\begin{tabular}{lcccc}
\hline & All patients & FAB-abnormal & FAB-normal & Mann-Whitney $U$ test \\
\hline No. of patients & 61 & 14 & 47 & $11: 36$ \\
Women : men & $19: 42$ & $8: 6$ & $52.5( \pm 10.5)$ & $\mathrm{P}=0.015$ \\
Age (years) & $54.5( \pm 11.3)$ & $61.1( \pm 11.7)$ & $15.0( \pm 7.1)$ & $\mathrm{P}=0.001$ \\
Disease duration (months) & $16.8( \pm 7.7)$ & $22.7( \pm 6.9)$ & $11.3( \pm 3.8)$ & $\mathrm{P}=0.175$ \\
Period of education (years) & $10.8( \pm 4.4)$ & $9.1( \pm 6.9)$ & $39.3( \pm 3.2)$ & $\mathrm{P}<0.001$ \\
ALSFRS-R & $38.4( \pm 3.5)$ & $35.1( \pm 2.7)$ & $27.5( \pm 1.8)$ & $P<0.001$ \\
K-MMSE & $26.5( \pm 3.2)$ & $23.0( \pm 4.5)$ & $16.72( \pm 1.12)$ & $P<0.001$ \\
Raw FAB & $15.34( \pm 3.26)$ & $10.71( \pm 3.83)$ & $16.31( \pm .84)$ & $P<0.001$ \\
Adjusted FAB & $15.15( \pm 3.77)$ & $11.24( \pm 3.40)$ & $2.98( \pm 0.15)$ & $P<0.001$ \\
$\quad$ Similarity & $2.72( \pm 0.73)$ & $1.86( \pm 1.17)$ & $2.26( \pm .74)$ & $P<0.001$ \\
$\quad$ Lexical fluency & $1.95( \pm 1.01)$ & $0.93( \pm 1.14)$ & $2.96( \pm 0.20)$ & $P=0.006$ \\
$\quad$ Motor Series & $2.82( \pm 0.62)$ & $2.36( \pm 1.15)$ & $2.87( \pm 0.34)$ & $P<0.001$ \\
$\quad$ Conflicting Instruction & $2.56( \pm 0.83)$ & $1.50( \pm 1.09)$ & $2.68( \pm 0.59)$ & $P<0.001$ \\
$\quad$ Go-No-Go task & $2.41( \pm 0.84)$ & $1.50( \pm 0.94)$ & $3.00( \pm .00)$ & $P=0.009$ \\
$\quad$ Prehension behavior & $2.90( \pm 0.54)$ & $2.57( \pm 1.09)$ & & $P=0.001$ \\
COWAT & & & $16.77( \pm 7.34)$ & $P=0.024$ \\
$\quad$ Phonemic Verbal fluency (s $+\mathrm{k})$ & $14.96( \pm 7.68)$ & $8.85( \pm 5.47)$ & $14.60( \pm 5.44)$ & $P=0.003$ \\
Phonemic written fluency (b+z) & $13.94( \pm 5.56)$ & $9.33( \pm 4.32)$ & $22.98( \pm 6.58)$ & $P=0.002$ \\
$\quad$ Semantic verbal fluency (animal+food) & $21.65( \pm 6.63)$ & $16.92( \pm 4.34)$ & $16.72( \pm 5.03)$ & $P=0.595$ \\
$\quad$ Semantic written fluency (flower+market) & $15.64( \pm 5.31)$ & $11.00( \pm 3.89)$ & $5.40( \pm 3.63)$ & \\
NPI & $5.31( \pm 3.69)$ & $5.00( \pm 3.98)$ & &
\end{tabular}

Data are expressed as mean values ( \pm standard deviation). Abbreviations : ALS; amyotrophic lateral sclerosis, FAB; frontal assessment battery, ALSFRS-R; ALS functional rating score revised, COWAT; controlled oral word association test, NPI; Neuropsychiatric inventory, K-MMSE; Mini-mental state exam Korean version. 
behavior, motor skills, and abstract reasoning. For instance, 59 patients $(96.7 \%)$ got a perfect score on the prehension behavior subtest. Frontal assessment battery-abnormal patients performed consistently worse than FAB-normal patients in the similarity, lexical fluency, go-no-go, and conflicting instruction tasks.

All of the seven patients (11.5\%) with general cognitive impairment (K-MMSE < 24) were FAB-abnormal. The FABnormal and FAB-abnormal patients performed significantly differently in all domains of the COWAT, regardless of whether they were spoken, written, phonological, or semantic. There was no difference in behavioral disturbance, as assessed by the NPI, between the two groups.

Frontal assessment battery scores were found to significantly correlate with both disease duration $(\rho=-0.324, P=0.011)$ and ALSFRS-R $(\rho=0.427, P=0.001)$ (Figure).

\section{Discussion}

The FAB test explores cognitive and behavioral domains under the control of the frontal lobes. ${ }^{15}$ Each of the six subtests is significantly correlated with medial and dorsolateral frontal lobe activity. ${ }^{16,17}$ Moreover, the Go-No-Go test is correlated with inferior prefrontal area. ${ }^{18}$ Here, the frequency of executive dysfunction documented by the FAB (23\%) was comparable to those reported in previous studies that used more extensive neuropsychological tests. ${ }^{1-3}$ This dysfunction was verified by a second test, the COWAT. Unfortunately, as there have been no reliable normal values of COWAT in the Korean population, we could not definitely classify the patients into normal or abnormal groups according to COWAT. In contrast to the relatively poor performance in the frontal executive function tests, the K-MMSE indicated that only a few patients had general cognitive dysfunction.

The FAB score was found to correlate significantly with both disease duration and ALSFRS-R. This suggests that frontal cognitive impairment may increase as the disease progresses, though a longitudinal study is required to confirm this. In previous studies of ALS patients, verbal fluency deficits were the most consistently reported cognitive impairment. Indeed, the patients in this study performed particularly poorly in the word generation subtest.

A NPI was used to evaluate neuropsychiatric problems common to patients with ALS, including depression, anxiety, altered eating habits, agitation, hallucination, abnormal sleep behavior, disinhibition, apathy, abnormal movement, mood elation, delusion, and stubbornness. ${ }^{19}$ Results from the FABnormal and FAB-abnormal groups were not statistically different, indicating that patients' psychiatric symptoms may be unrelated to the cognitive impairment documented by the FAB. The relation between behavioral disturbances and cognitive impairments in ALS remains to be elucidated in future studies.

The drawback of using the FAB to evaluate frontal lobe dysfunction in ALS patients is that performance may be affected by motor impairments such as arm weakness and speech disturbance.$^{20}$ Lexical fluency, in particular, could be affected by dysarthria in ALS patients with bulbar involvement, and the Luria's motor series task could be affected by hand weakness. Thus, even if we excluded those patients who have either severe arm weakness ( $\leq$ MRC grade III) or speech impairment (where speech is intelligible only after repetition), a possible confounding effect of motor impairment cannot be ruled out. Thus, further study is required to assess how FAB performance decreases as frontal cognitive impairment increases in patients with advanced ALS; this information will be particularly useful for evaluating the results of longitudinal studies. In addition, this study does have some limitations. For instance, the methods and cutoff points were based on those of an Italian normative study, but were applied to a Korean population. However, linguistic and cultural differences between these two populations may make extrapolations difficult or inaccurate. Additionally, we were unable to collect longitudinal data, which could further explore the correlation between cognitive dysfunction and both disease duration and severity. Future studies in Korean populations should examine larger study groups and should combine FAB with genetic research, hypoventilation, and brain imaging in order to more accurately measure the prevalence of FTD in ALS.

\section{Conclusions}

Despite the need for further investigations of FAB, it has several clear advantages over more extensive neuropsychological tests. It is a time-efficient and cost-saving method that does not require highly skilled techniques, yet produces results similar to those of other, more extensive, neuropsychological studies. Thus, we suggest that FAB is an effective and reliable screening method for assessing frontal lobe dysfunction in ALS patients.

\section{ACKNOWLEDGMENT}

This study was supported by a grant of the Korea Healthcare technology Research and Development Project, Ministry for Health, Welfare and Family Affairs, Republic of Korea (A091049) and a grant of the Seoul National University Hospital (03-2005-0220). 


\section{REFERENCES}

1. Ringholz GM, Appel SH, Bradshaw M, Cooke NA, Mosnik DM, Schulz PE. Prevalence and patterns of cognitive impairment in sporadic ALS. Neurology. 2005;65:586-90.

2. Murphy JM, Henry RG, Langmore S, Kramer JH, Miller BL, Lomen-Hoerth C. Continuum of frontal lobe impairment in amyotrophic lateral sclerosis. Arch Neurol. 2007;64:530-4.

3. Strong MJ, Grace GM, Freedman M, et al. Consensus criteria for the diagnosis of frontotemporal cognitive and behavioural syndromes in amyotrophic lateral sclerosis. Amyotroph Lateral Scler. 2009;10:131-46.

4. Olney RK, Murphy J, Forshew D, et al. The effects of executive and behavioral dysfunction on the course of ALS. Neurology. 2005; 65:1774-7.

5. Dubois B, Slachevsky A, Litvan I, Pillon B. The FAB: a Frontal Assessment Battery at bedside. Neurology. 2000;50:1621-6.

6. Appollonio I, Leone M, Isella V, et al. The Frontal Assessment Battery (FAB): normative values in an Italian population sample. Neurol Sci. 2005;26:108-16.

7. Lima CF, Meireles LP, Fonseca R, Castro SL, Garrett C. The Frontal Assessment Battery (FAB) in Parkinson's disease and correlations with formal measures of executive functioning. J Neurol. 2008;255:1756-61.

8. Slachevsky A, Villalpando JM, Sarazin M, Hahn-Barma V, Pillon B, Dubois B. Frontal assessment battery and differential diagnosis of frontotemporal dementia and Alzheimer disease. Arch Neurol. 2004;61:1104-7.

9. Brooks BR, Miller RG, Swash M, Munsat TL. World Federation of Neurology Research Group on Motor Neuron Disease. El Escorial revisited: revised criteria for the diagnosis of amyotrophic lateral sclerosis. Amyotroph Lateral Scler. 2000;1: 293-8.

10. Cedarbaum JM, Stambler N, Malta E, et al. The ALSFRS-R: a revised ALS functional rating scale that incorporates assessments of respiratory function. BDNF ALS Study Group (Phase III). J Neurol Sci. 1999;169:13-21.
11. Rodriguez-Aranda C, Martinussen M. Age-related differences in performance of phonemic verbal fluency measured by Controlled Oral Word Association Task (COWAT): a metaanalytic study. Dev Neuropsychol. 2006;30:697-717.

12. Lee BR, Chun JE, Lee SJ, Lee BH, Yu IK, Kim SY. Cognitive dysfunction in 16 Patients with carotid stenosis: detailed neuropsychological findings. J Clin Neurol. 2007;3:9-17.

13. Han C, Jo SA, Jo I, Kim E, Park MH, Kang Y. An adaptation of the Korean mini-mental state examination (K-MMSE) in elderly Koreans: demographic influence and population-based norms (the AGE study). Arch Gerontol Geriatr. 2008;47:302-10.

14. Blair M, Kertesz A, Davis-Faroque N, et al. Behavioral measures in frontotemporal lobar dementia and other dementias: the utility of the frontal behavioural inventory and the neuropsychiatric inventory in a national cohort study. Dement Geriatr Cogn Disord. 2007;23:406-15.

15. Kim TH, Huh Y, Choe JY, et al. Korean version of frontal assessment battery: psychometric properties and normative data. Dement Geriatr Cogn Disord. 2010;29:363-70.

16. Guedj E, Allali G, Goetz C, et al. Frontal Assessment Battery is a marker of dorsolateral and medial frontal functions: a SPECT study in frontotemporal dementia. J Neurol Sci. 2008;273:84-7.

17. Warburton E, Wise RJ, Price CJ, et al. Noun and Verb retrieval by normal subjects. Study with PET. Brain. 1996;119:159-79.

18. Konish S, Nakajima K, Uchida I, Kikyo H, Kameyama M, Miyashita Y. Common inhibitory mechanism in human inferior prefrontal cortex revealed by event-related functional MRI. Brain. 1999;122:981-91.

19. Elman LB, Grossman M. Neuropsychiatric features of amyotrophic lateral sclerosis. Neuro Rehab. 2007;22:425-9.

20. Oskarsson B, Quan D, Rollins YD, Neville HE, Ringel SP, Arciniegas DB. Using the Frontal Assessment Battery to identify executive function impairments in amyotrophic lateral sclerosis: a preliminary experience. Amyotroph Lateral Scler. 2010;11: 244-7. 